\title{
Schedulability Analysis and Optimization for the Synthesis of Multi-Cluster Distributed Embedded Systems
}

Pop, Paul; Eles, Petru; Peng, Zebo

Published in:

IEE Proceedings - Computers and digital Techniques

Link to article, DOI:

10.1049/ip-cdt:20030829

Publication date:

2003

Document Version

Publisher's PDF, also known as Version of record

Link back to DTU Orbit

Citation (APA):

Pop, P., Eles, P., \& Peng, Z. (2003). Schedulability Analysis and Optimization for the Synthesis of Multi-Cluster Distributed Embedded Systems. IEE Proceedings - Computers and digital Techniques, 150(5), 303-312. https://doi.org/10.1049/ip-cdt:20030829

\section{General rights}

Copyright and moral rights for the publications made accessible in the public portal are retained by the authors and/or other copyright owners and it is a condition of accessing publications that users recognise and abide by the legal requirements associated with these rights.

- Users may download and print one copy of any publication from the public portal for the purpose of private study or research.

- You may not further distribute the material or use it for any profit-making activity or commercial gain

- You may freely distribute the URL identifying the publication in the public portal 


\title{
Schedulability analysis and optimisation for the synthesis of multi-cluster distributed embedded systems
}

\author{
P. Pop, P. Eles and Z. Peng
}

\begin{abstract}
An approach to schedulability analysis for the synthesis of multi-cluster distributed embedded systems consisting of time-triggered and event-triggered clusters, interconnected via gateways, is presented. A buffer size and worst case queuing delay analysis for the gateways, responsible for routing inter-cluster traffic, is also proposed. Optimisation heuristics for the priority assignment and synthesis of bus access parameters aimed at producing a schedulable system with minimal buffer needs have been proposed. Extensive experiments and a real-life example show the efficiency of the approaches.
\end{abstract}

\section{Introduction}

Depending on the particular application, an embedded system has certain requirements for performance, cost, dependability, size etc. For hard real-time applications the timing requirements are extremely important. Hence, in order to function correctly, an embedded system implementing such an application has to meet its deadlines.

Process scheduling and schedulability analysis have been intensively studied for the past decades $[1,2]$. There are two basic approaches for handling tasks in real-time applications [3]. In the event-triggered approach (ET), activities are initiated whenever a particular event is noted. In the timetriggered (TT) approach, activities are initiated at predetermined points in time. There has been a long debate in the real-time and embedded systems communities concerning the advantages of each approach [3-5]. Several aspects have been considered in favour of one or the other approach, such as flexibility, predictability, jitter control, processor utilisation, testability etc.

The same duality is reflected at the level of the communication infrastructure, where communication activities can be triggered either dynamically, in response to an event, as with the controller area network (CAN) bus [6], or statically, at predetermined moments in time, as in the case of time-division multiple access (TDMA) protocols and, in particular, the time-triggered protocol (TTP) [3].

A few approaches have been proposed for the schedulability analysis of distributed real-time systems, taking into consideration both process and communication scheduling. In $[7,8]$ Tindell et al. provided a framework for the analysis of ET process sets interconnected through an infrastructure based on either the CAN protocol or a generic TDMA protocol. In $[9,10]$ we have developed an analysis allowing

(C) IEE, 2003

IEE Proceedings online no. 20030829

doi: 10.1049/ip-cdt:20030829

Paper received 16th May 2003

The authors are with the Department of Computer and Information Science, Linköping University, SE-581 83, Linköping, Sweden for either TT or ET process sets communicating over the TTP.

An interesting comparison of the ET and TT approaches, from a more industrial, in particular automotive, perspective, can be found in [11]. The conclusion there is that one has to choose the right approach depending on the particularities of the processes. This means not only that there is no single 'best' approach to be used, but also that inside a certain application the two approaches can be used together, some tasks being TT and others ET. The fact that such an approach is suitable for automotive applications is demonstrated by the following two trends, which are currently considered to be of foremost importance not only for the automotive industry, but also for other categories of industrial applications:

1 The development of bus protocols that support both static and dynamic communication [12]. This allows for ET and TT processes to share the same processor as well as dynamic and static communications to share the same bus. In [13] we have addressed the problem of timing analysis for such systems.

2 Complex systems are designed as interconnected clusters of processors. Each such cluster can be either TT or ET. In a time-triggered cluster (TTC), processes and messages are scheduled according to a static cyclic policy, with the bus implementing the TTP. In an event-triggered cluster (ETC), the processes are scheduled according to a priority based pre-emptive approach, while messages are transmitted using the priority-based CAN protocol. Depending on their particular nature, certain parts of an application can be mapped on processors belonging to an ETC or a TTC. The critical element of such an architecture is the gateway, which is a node connected to both types of clusters, and is responsible for the inter-cluster routing of hard real-time traffic.

In this paper we propose an approach to schedulability analysis for the synthesis of multi-cluster distributed embedded systems, including also buffer need analysis and worst case queuing delays of inter-cluster traffic. We have also developed optimisation heuristics for the synthesis of bus access parameters as well as process and 
message priorities aimed at producing a schedulable system such that buffer sizes are minimised.

Efficient implementation of new, highly sophisticated automotive applications entails the use of TT process sets together with ET sets implemented on top of complex distributed architectures. In this context, this paper is the first to address the analysis and optimisation of heterogeneous TT and ET systems implemented on multi-cluster embedded networks.

\section{Application model and system architecture}

\subsection{Application model}

We model an application $\Gamma$ as a set of process graphs $G_{i} \in \Gamma$ (Fig. 1). Nodes in the graph represent processes and arcs represent dependency between the connected processes. The communication time between processes mapped on the same processor is considered to be part of the process worst case execution time and is not modelled explicitly. Communication between processes mapped to different processors is preformed by message passing over the buses and, if needed, through the gateway. Such message passing is modelled as a communication process inserted on the arc connecting the sender and the receiver process (the black dots in Fig. 1).

Each process $P_{i}$ is mapped on a processor processor $_{P_{i}}$ (mapping represented by shading in Fig. 1), and has a worst case execution time $C_{i}$ on that processor (depicted to the left of each node). The size of each message is known (in bytes, indicated to its left), and also its period, which is identical to that of the sender process. Processes and messages activated based on events also have a uniquely assigned priority, priority $_{P_{i}}$ for processes and priority $m_{m_{i}}$ for messages.

All processes and messages belonging to a process graph $G_{i}$ have the same period $T_{i}=T_{G_{i}}$, which is the period of the process graph. A deadline $D_{G_{i}} \leq T_{G_{i}}$ is imposed on each process graph $G_{i}$. Deadlines can also be placed locally on processes. If communicating processes are of different periods, they are combined into a hypergraph capturing all process activations for the hyperperiod (lowest common multiple of all periods).

\subsection{Hardware architecture}

We consider architectures consisting of several clusters, interconnected by gateways (Fig. 2 depicts a two-cluster example). A cluster is composed of nodes which share a broadcast communication channel. Every node consists, among others, of a communication controller and a CPU. The gateways, connected to both types of clusters, have two communication controllers, for TTP and CAN. The communication controllers implement the protocol services, and run independently of the node's CPU. Communication
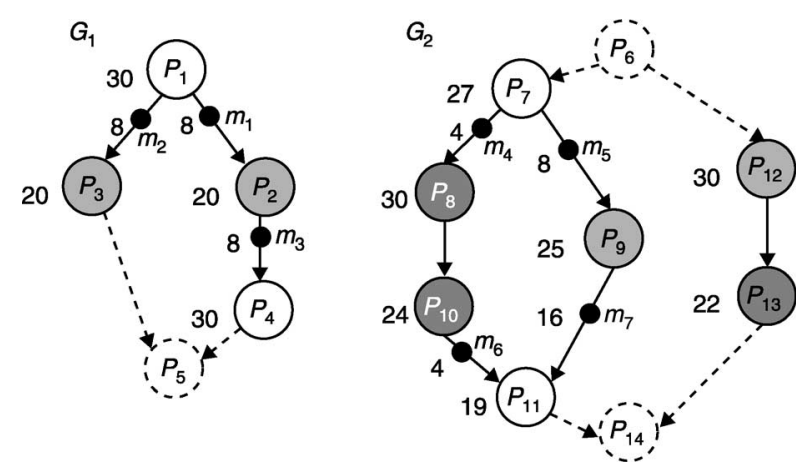

Fig. 1 Application model example

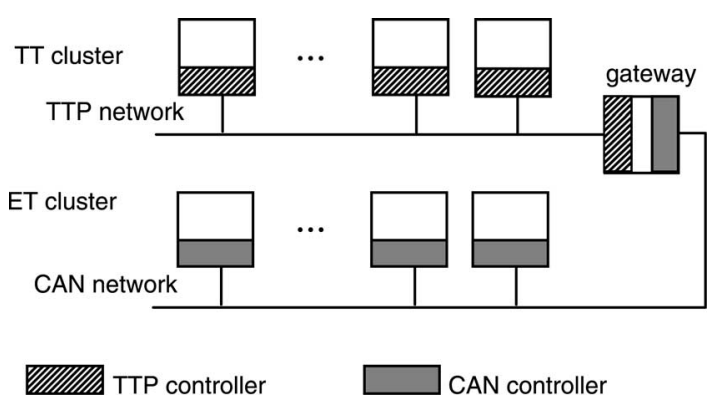

Fig. 2 System architecture example

with the CPU is performed through a message base interface (MBI), which is usually implemented as a dual ported RAM (Fig. 3).

Communication between the nodes on a TTC is based on the TTP [3]. The bus access scheme is TDMA, where each node $N_{i}$, including the gateway node, can transmit only during a predetermined time interval, the so-called TDMA slot $S_{i}$. In such a slot, a node can send several messages packed in a frame. A sequence of slots corresponding to all the nodes in the TTC is called a TDMA round. A node can have only one slot in a TDMA round. Several TDMA rounds can be combined together in a cycle that is repeated periodically. The TDMA access scheme is imposed by a message descriptor list (MEDL) that is located in every TTP controller. The MEDL serves as a schedule table for the TTP controller, which has to know when to send/receive a frame to/from the communication channel.

On an ETC the CAN [6] protocol is used for communication. The CAN bus is a priority bus that employs a collision avoidance mechanism, whereby the node that transmits the message with the highest priority wins the contention. Message priorities are unique and are encoded in the frame identifiers, which are the first bits to be transmitted on the bus.

\subsection{Software architecture}

A real-time kernel is responsible for the activation of processes and transmission of messages on each node. On a TTC, the processes are activated based on the local schedule tables, and messages are transmitted according to the MEDL. On an ETC, we have a scheduler that decides on the activation of ready processes and transmission of messages, based on their priorities.

Figure 3 illustrates the message passing mechanism. Here we concentrate on the communication between processes located on different clusters. For message passing details within a TTC the reader is directed to [14], while the infrastructure needed for communications on an ETC has been detailed in [7].

Consider the example in Fig. 3, where the process graph $G_{1}$ from Fig. 1 is mapped on to the two clusters. Processes $P_{1}$ and $P_{4}$ are mapped on node $N_{1}$ of the TTC, while $P_{2}$ and $P_{3}$ are mapped on node $N_{2}$ of the ETC. Process $P_{1}$ sends messages $m_{1}$ and $m_{2}$ to processes $P_{2}$ and $P_{3}$, respectively, while $P_{2}$ sends message $m_{3}$ to $P_{4}$.

The transmission of messages from the TTC to the ETC takes place in the following way (see Fig. 3). $P_{1}$, which is statically scheduled, is activated according to the schedule table, and when it finishes it calls the send kernel function in order to send $m_{1}$ and $m_{2}$, indicated in the Figure by a circled number (1). Messages $m_{1}$ and $m_{2}$ have to be sent from node $N_{1}$ to node $N_{2}$. At a certain time, known from the schedule table, the kernel transfers $m_{1}$ and $m_{2}$ to the TTP controller by packaging them into a frame in the MBI. 


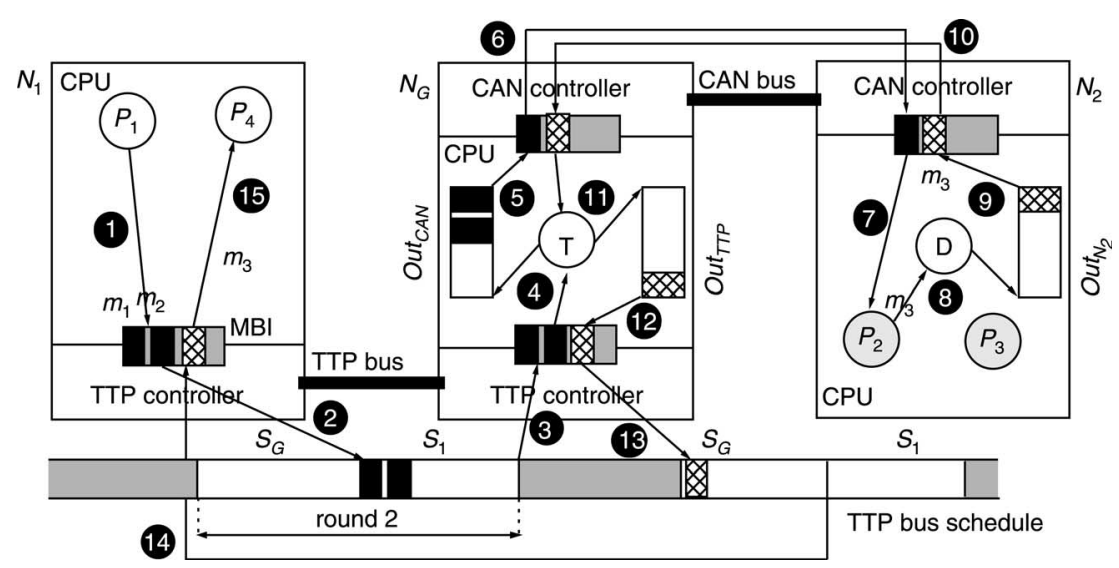

Fig. 3 Message passing example

Later on, the TTP controller knows from its MEDL when it has to take the frame from the MBI, in order to broadcast it on the bus. In our example, the timing information in the schedule table of the kernel and the MEDL is determined in such a way that the broadcasting of the frame is done in the slot $S_{1}$ of round two (2). The TTP controller of the gateway node $N_{G}$ knows from its MEDL that it has to read a frame from slot $S_{1}$ of a round two and to transfer it into its MBI (3). Invoked periodically, having the highest priority on node $N_{G}$, and with a period which guarantees that no messages are lost, the gateway process $T$ copies messages $m_{1}$ and $m_{2}$ from the MBI to the TTP-to-CAN priority-ordered message queue $\mathrm{Out}_{C A N}$ (4). The highest priority message in the queue, in our case $m_{1}$, will tentatively be broadcast on the CAN bus (5. Whenever message $m_{1}$ is the highest priority message on the CAN bus, it will be successfully broadcast and will be received by the interested nodes, in our case node $N_{2}$ (6). The CAN communication controller of node $N_{2}$ receiving $m_{1}$ will copy it in the transfer buffer between the controller and the CPU, and raise an interrupt that will activate a delivery process, responsible for activating the corresponding receiving process, in our case $P_{2}$, and hand over message $m_{1}$ that finally arrives at the destination (7).

Message $m_{3}$ (depicted in Fig. 3 as a hashed rectangle) sent by process $P_{2}$ from the ETC will be transmitted to process $P_{4}$ on the TTC. The transmission starts when $P_{2}$ calls its send function and enqueues $m_{3}$ in the priority-ordered Out $_{N_{2}}$ queue (8. When $m_{3}$ has the highest priority on the bus, it will be removed from the queue (9) and broadcast on the CAN bus (10, arriving at the gateway's CAN controller where it raises an interrupt. Based on this interrupt, the gateway transfer process $T$ is activated, and $m_{3}$ is placed in the Out $_{T T P}$ FIFO queue (11. The gateway node $N_{G}$ is only able to broadcast on the TTC in the slot $S_{G}$ of the TDMA rounds circulating on the TTP bus. According to the MEDL of the gateway, a set of messages not exceeding size $S_{S_{G}}$ of the slot $S_{G}$ will be removed from the front of the $O u t_{T T P}$ queue in every round, and packed in the $S_{G}$ slot (18. Once the frame is broadcast (18) it will arrive at node $N_{1}$ (14), where all the messages in the frame will be copied in the input buffers of the destination processes (1). Process $P_{4}$ is activated according to the schedule table, which has to be constructed so that it accounts for the worst-case communication delay of message $m_{3}$, bounded by the analysis in Section 4, and thus when $P_{4}$ starts executing it will find $m_{3}$ in its input buffer.

As part of our timing analysis and synthesis approach, all the local schedule tables and MEDLs are generated on the TTC, and the message and process priorities for the activities are generated on the ETC, as well as buffer sizes and bus configurations so that the global system is schedulable.

\section{Problem formulation}

As input to our problem we have an application $\Gamma$ given as a set of process graphs mapped on an architecture consisting of a TTC and an ETC interconnected through a gateway.

We are interested first in finding a system configuration denoted by a 3-tuple $\Psi=\langle\phi, \beta, \pi\rangle$ such that the application $\Gamma$ is schedulable. Determining a system configuration $\Psi$ means deciding on:

- The set $\phi$ of the offsets corresponding to each process and message in the system (see Section 4). The offsets of processes and messages on the TTC practically represent the local schedule tables and MEDLs.

- The TTC bus configuration $\beta$, indicating the sequence and size of the slots in a TDMA round on the TTC.

- The priorities of the processes and messages on the ETC, captured by $\pi$.

Once a configuration leading to a schedulable application is found, we are interested in finding a system configuration that minimises the total queue sizes needed to run a schedulable application. The approach presented in this paper can be easily extended to cluster configurations where there are several ETCs and TTCs interconnected by gateways.

Consider the example in Fig. 4, where there is shown the process graph $G_{1}$ from Fig. 1 mapped on the twocluster system, as indicated in Fig. 3. In the system configuration of Fig. $4 a$ we consider that, on the TTP bus, the gateway transmits in the first slot $\left(S_{G}\right)$ of the TDMA round, while node $N_{1}$ transmits in the second slot $\left(S_{1}\right)$. The priorities inside the ETC have been set such that priority $_{m_{1}}>$ priority $_{m_{2}}$ and priority $_{P_{3}}>$ priority $_{P_{2}}$. In such a setting, $G_{1}$ will miss its deadline, which was set at $200 \mathrm{~ms}$. However, changing the system configuration as in Fig. $4 b$, so that slot $S_{1}$ of $N_{1}$ comes first, we are able to send $m_{1}$ and $m_{2}$ sooner, and thus reduce the response time and meet the deadline. The response times and resource usage do not, of course, depend only on the TDMA configuration. In Fig. $4 c$, for example, we have modified the priorities of $P_{2}$ and $P_{3}$ so that $P_{2}$ is the higher priority process. In such a situation, $P_{2}$ is not interrupted when the delivery of message $m_{2}$ is supposed to activate $P_{3}$ and, thus, eliminating the interference, we are able to meet the deadline, even with the TTP bus configuration of Fig. $4 a$. 

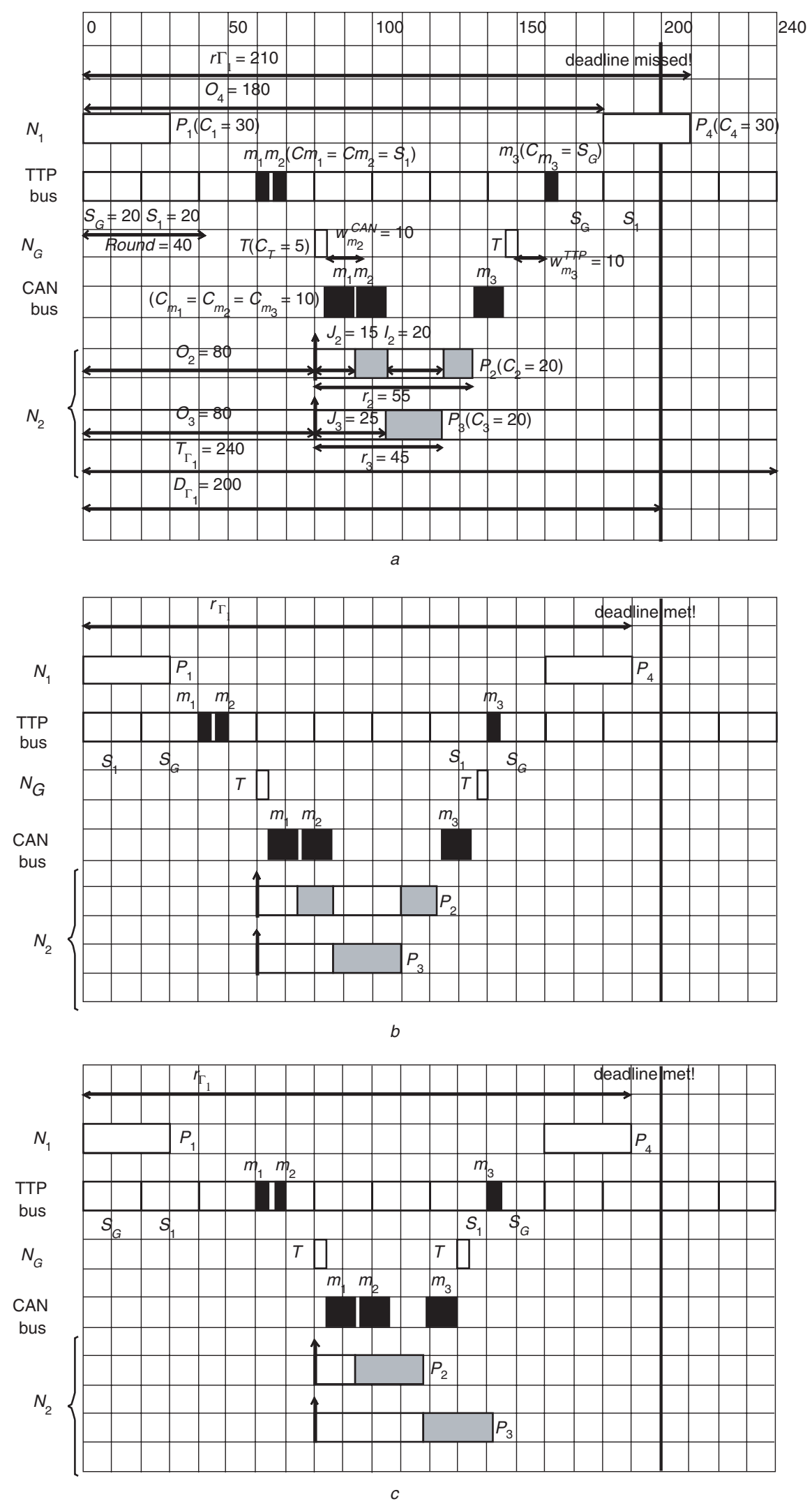

Fig. 4 Scheduling examples

a $G_{1}$ misses its deadline

b $S_{1}$ is the first slot, $m_{1}, m_{2}$ are sent sooner, $G_{1}$ meets its deadline

c $P_{2}$ is the high priority process on $N_{2}, G_{1}$ meets its deadline

\section{Multi-cluster scheduling}

In this Section we propose an analysis for hard real-time applications mapped on multi-cluster systems. The aim of such an analysis is to find out if a system is schedulable,

i.e. if all the timing constraints are met. In addition, we are also interested in bounding the queue sizes.

In a TTC an application is schedulable if it is possible to build a schedule table such that the timing requirements are satisfied. In a ETC, the answer to whether or 
not a system is schedulable is given by a schedulability analysis.

In this paper, for the ETC we use a response time analysis, where the schedulability test consists of the comparison between the worst-case response time $r_{i}$ of a process $P_{i}$ and its deadline $D_{i}$. Response time analysis of data dependent processes with static priority pre-emptive scheduling has been proposed in [15-17], and has been also extended to consider the CAN protocol [7]. The authors use the concept of offset to handle data dependencies. Thus each process $P_{i}$ is characterised by an offset $O_{i}$, measured from the start of the process graph, that indicates the earliest possible start time of $P_{i}$. For example, in Fig. $4 a, O_{2}=80$, as process $P_{2}$ cannot start before receiving $m_{1}$, which is available at the end of slot $S_{1}$ in round two. The same is true for messages, their offset indicating the earliest possible transmission time.

Determining the schedulability of an application mapped on a multi-cluster system cannot be addressed separately for each type of cluster, as the inter-cluster communication creates a circular dependency: the static schedules determined for the TTC influence through the offsets the response times of the processes on the ETC, which in their turn influence the schedule table construction in the TTC. In Fig. $4 a$ placing $m_{1}$ and $m_{2}$ in the same slot leads to equal offsets for $P_{2}$ and $P_{3}$. Because of this, $P_{3}$ will interfere with $P_{2}$ (which would not be the case if $m_{2}$ sent to $P_{3}$ to be scheduled in round four) and thus the placement of $P_{4}$ in the schedule table has to be accordingly delayed to guarantee the arrival of $m_{3}$.

In our response time analysis we consider the influence between the two clusters by making the following observations:

- The start time of process $P_{i}$ in a schedule table on the TTC is its offset $O_{i}$.

- The worst-case response time $r_{i}$ of a TT process is its worst-case execution time, i.e. $r_{i}=C_{i}$ (TT processes are not pre-emptable).

- The response times of the messages exchanged between two clusters have to be calculated according to the schedulability analysis described in Section 4.1.

- The offsets have to be set by a scheduling algorithm so that the precedence relationships are preserved. This means that, if process $P_{B}$ depends on process $P_{A}$, the following condition must hold: $O_{B} \geq O_{A}+r_{A}$. Note that, for the processes on a TTC receiving messages from the ETC, this translates to setting the start times of the processes so that a process is not activated before the worst-case arrival time of

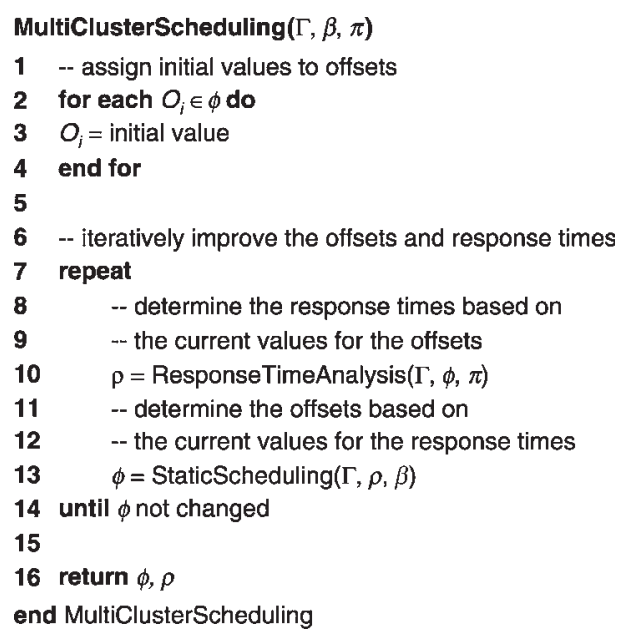

Fig. 5 MultiClusterScheduling algorithm the message from the ETC. In general, offsets on the TTC are set such that all the necessary messages are present at the process invocation.

The MulticlusterScheduling algorithm in Fig. 5 receives as input the application $\Gamma$, the TTC bus configuration $\beta$ and the ET process and message priorities $\pi$, and produces the offsets $\phi$ and response times $\rho$. The algorithm starts by assigning to all offsets an initial value obtained by a static scheduling algorithm applied on the TTC without considering the influence from the ETC (lines 2-4). The response times of all processes and messages in the ETC are then calculated according to the analysis in Section 4.1 by using the ResponseTimeAnalysis function (line 10). Based on the response times, offsets of the TT processes can be defined such that all messages received from the ETC cluster are present at process invocation. Considering these offsets as constraints, a static scheduling algorithm can derive the schedule tables and MEDLs of the TTC cluster (line 13). For this purpose we use the list scheduling based approach presented in [9]. Once new values have been determined for the offsets, they are fed back to the response time calculation function, thus obtaining new, tighter (i.e. smaller, less pessimistic) values for the worst-case response times. The algorithm stops when the response times cannot be further tightened and consequently the offsets remain unchanged. Termination is guaranteed if processor and bus loads are smaller than $100 \%$ (see Section 4.2) and deadlines are smaller than the periods.

\subsection{Schedulability and resource analysis}

The analysis in this Section is used in the ResponseTimeAnalysis function in order to determine the response times for processes and messages on the ETC. It receives as input the application $\Gamma$, the offsets $\phi$ and the priorities $\pi$, and it produces the set $\rho$ of worst case response times.

We have extended the framework provided by $[7,16]$ for an ETC. Thus the response time of a process $P_{i}$ on the ETC is:

$$
r_{i}=J_{i}+w_{i}+C_{i}
$$

where $J_{i}$ is the jitter of process $P_{i}$ (the worst-case delay between the activation of the process and the start of its execution), and $C_{i}$ is its worst-case execution time. The interference $w_{i}$ from other processes running on the same processor is given by:

$$
w_{i}=B_{i}+\sum_{\forall p_{j} \in h p\left(P_{i}\right)}\left\lceil\frac{w_{i}+J_{j}-O_{i j}}{T_{j}}\right\rceil_{0} C_{j}
$$

In (2), the blocking factor $B_{i}$ represents interference from lower priority processes that are in their critical section and cannot be interrupted. The second term captures the interference from higher priority processes $P_{j} \in h p\left(P_{i}\right)$, where $O_{i j}$ is a positive value representing the relative offset of process $P_{j}$ to $P_{i}$. The $\lceil x\rceil_{0}$ operator is the positive ceiling, which returns the smallest integer greater than $x$, or 0 if $x$ is negative.

The same analysis (1) and (2) can be applied for messages on the CAN bus:

$$
r_{m}=J_{m}+w_{m}+C_{m}
$$

where $J_{m}$ is the jitter of message $m$, which in the worst case is equal to the response time $r_{S(m)}$ of the sender process $P_{S(m)}, w_{m}$ is the worst-case queuing delay experienced by $m$ at the communication controller, and $C_{m}$ is the worst-case time it takes for a message $m$ to reach the destination controller. In CAN, $C_{m}$ depends on the frame configuration 
and message size $s_{m}$, while in TTP it is equal to the slot size where $m$ is transmitted.

The response time analysis for processes and messages are combined by realising that the jitter of a destination process depends on the communication delay between sending and receiving a message. Thus, for a process $P_{D(m)}$ that receives a message $m$ from a sender process $P_{S(m)}$, the release jitter is $J_{D(m)}=r_{m}$.

The worst-case queueing delay for a message ( $w_{m}$ in (3)) is calculated differently depending on the type of message passing employed:

1 From an ETC node to another ETC node (in which case $w_{m}^{N_{i}}$ represents the worst-case time a message $m$ has to spend in the $O u t_{N_{i}}$ queue on ETC node $N_{i}$ ). An example of such a message is $m_{3}$ in Fig. $4 a$, which is sent from the ETC node $N_{3}$ to the gateway node $N_{G}$.

2 From a TTC node to an ETC node ( $w_{m}^{C A N}$ is the worst-case time a message $m$ has to spend in the $O u t_{C A N}$ queue). In Fig. $4 a$, message $m_{1}$ is sent from the TTC node $N_{1}$ to the ETC node $N_{2}$.

3 From an ETC node to a TTC node (where $w_{m}^{T T P}$ captures the time $m$ has to spend in the $O u t_{T T P}$ queue). Such message passing happens in Fig. $4 a$, where message $m_{3}$ is sent from the ETC node $N_{3}$ to the TTC node $N_{1}$ through the gateway node $N_{G}$ where it has to wait for a time $w_{m}^{T T P}$ in the Out $t_{T T P}$ queue.

The messages sent from a TTC node to another TTC node are taken into account when determining the offsets (StaticScheduling, Fig. 5), and hence are not involved directly in the ETC analysis.

We next show how the worst queueing delays and the bounds on the queue sizes are calculated for each of the previous three cases.

4.1.1 From ETC to ETC and from TTC to ETC: The analyses for $w_{m}^{N_{i}}$ and $w_{m}^{C A N}$ are similar. Once $m$ is the highest priority message in the $\mathrm{Out}_{\text {CAN }}$ queue, it will be sent by the gateway's CAN controller as a regular CAN message, therefore the same equation for $w_{m}$ can be used:

$$
w_{m}=B_{m}+\sum_{\forall m_{j} \in h p(m)}\left\lceil\frac{w_{m}+J_{j}-O_{m j}}{T_{j}}\right\rceil_{0} C_{j}
$$

Intuition tells us that $m$ has to wait, in the worst case, first for the largest lower priority message that is just being transmitted $\left(B_{m}\right)$ as well as for the higher priority $m_{j} \in$ $h p(m)$ messages that have to be transmitted ahead of $m$ (the second term). In the worst case, the time it takes for the largest lower priority message $m_{k} \in l p(m)$ to be transmitted to its destination is:

$$
B_{m}=\max _{\forall m_{k} \in l p(m)}\left(C_{k}\right)
$$

Note that in our case, $l p(m)$ and $h p(m)$ also include messages produced by the gateway node, transferred from the TTC to the ETC.

We are also interested to bound the size $s_{m}^{C A N}$ of the Out $_{C A N}$ and $s_{m}^{N_{i}}$ of the Out $N_{i}$ queue. In the worst case, message $m$, all the messages with higher priority than $m$ will be in the queue, awaiting transmission. Summing up their sizes, and finding out what is the most critical instant, we get the worst-case queue size:

$$
s_{\text {Out }}=\max _{\forall m}\left(s_{m}+\sum_{\forall m_{j} \in h p(m)}\left\lceil\frac{w_{m}+J_{j}-O_{m j}}{T_{j}}\right\rceil_{0} s_{j}\right)
$$

where $s_{m}$ and $s_{j}$ are the sizes of message $m$ and $m_{j}$, respectively.

4.1.2 From ETC to TTC: The time a message $m$ has to spend in the Out $_{T T P}$ queue in the worst case depends on the total size of messages queued ahead of $m$ (Out $_{\text {TTP }}$ is a FIFO queue), the size $S_{G}$ of the gateway slot responsible for carrying the CAN messages on the TTP bus, and the frequency $T_{T D M A}$ with which this slot $S_{G}$ is circulating on the bus:

$$
w_{m}^{T T P}=B_{m}+\left\lceil\frac{S_{m}+I_{m}}{S_{G}}\right\rceil T_{T D M A}
$$

where $I_{m}$ is the total size of the messages queued ahead of $m$. Those messages $m_{j} \in h p(m)$ are ahead of $m$, which have been sent from the ETC to the TTC, and have higher priority than $m$ :

$$
I_{m}=\sum_{\forall m_{j} \in h p(m)}\left\lceil\frac{w_{m}^{T T P}+J_{m}-O_{m j}}{T_{j}}\right\rceil_{0} s_{j}
$$

where the message jitter $J_{m}$ is in the worst case the response time of the sender process, $J_{m}=r_{S(m)}$.

The blocking factor $B_{m}$ is the time interval in which $m$ cannot be transmitted because the slot $S_{G}$ of the TDMA round has not arrived yet, and is determined as:

$$
T_{T D M A}-O_{m} \bmod T_{T D M A}+O_{S_{G}}
$$

where $O_{S_{G}}$ is the offset of the gateway slot in a TDMA round.

Determining the size of the queue needed to accommodate the worst case burst of messages sent from the CAN cluster is done by finding out the worst instant of the following sum:

$$
s_{\text {Out }}^{T T P}={ }_{\forall m}^{\max }\left(s_{m}+I_{m}\right)
$$

\subsection{Response time analysis example}

Figure 6 presents the equations for the system in Fig. $4 a$. The jitter of $P_{2}$ depends on the response time of the gateway transfer process $T$ and the response time of message $m_{1}$, $J_{2}=r_{m_{1}}$. Similarly, $J_{3}=r_{m_{2}}$. We have noted that $J_{m_{1}}=$ $J_{m_{2}}=r_{T}$. The response time $r_{m_{3}}$ denotes the response time of $m_{3}$ sent from process $P_{2}$ to the gateway process $T$, while $r_{m_{3}^{\prime}}$ is the response time of the same message $m_{3}$ sent now from $T$ to $P_{4}$.

The equations are recurrent, and they will converge if the processor and bus utilisation are under $100 \%$ [8].

$$
\begin{aligned}
& \left.\begin{array}{l}
r_{2}=J_{2}+w_{2}+C_{2}, w_{2}=B_{2}+\left\lceil\frac{w_{2}+J_{3}-O_{2,3}}{T}\right. \\
r_{3}=J_{3}+w_{3}+C_{3}, w_{3}=B_{3}
\end{array}\right\rceil C_{3} \\
& \begin{array}{l}
r_{m_{1}}=J_{m_{1}}+w_{m_{1}}^{C A N}+C_{m_{1}}, w_{m_{1}}^{C A N}=B_{m_{1}} \\
r_{m_{2}}=J_{m_{2}}+w_{m_{2}}^{C A N}+C_{m_{2}}, w_{m_{2}}^{C A N}=B_{m_{2}}+\left\lceil\frac{w_{m_{2}}+J_{m_{1}}-O_{m_{2}, m_{1}}}{T}\right\rceil C_{3}
\end{array} \\
& r_{m_{3}}=J_{m_{3}}+w_{m_{3}}^{N_{2}}+C_{m_{3}} \\
& r_{m_{3^{\prime}}}=J_{m_{3^{\prime}}}+W_{m_{3^{\prime}}}^{T T P}+C_{m_{3},}, w_{m_{3^{\prime}}}^{T T P}=B_{m_{3^{\prime}}}+\left\lceil\frac{S_{m_{3}}}{T}\right\rceil T_{T D M A^{\prime}} \\
& B_{m_{3}{ }^{\prime}}=T_{T D M A} O_{m_{3}} \bmod T_{T D M A}+O_{S_{G}}
\end{aligned}
$$

Fig. 6 Response time analysis example

IEE Proc.-Comput. Digit. Tech., Vol. 150, No. 5, September 2003 
Considering a TDMA round of $40 \mathrm{~ms}$, with two slots each of $20 \mathrm{~ms}$ as in Fig. $4 a, r_{T}=5 \mathrm{~ms}, 10 \mathrm{~ms}$ for the transmission times on CAN for $m_{1}$ and $m_{2}$, and using the offsets in the Figure, the equations will converge to the values indicated in Fig. $4 a$ (all values are in milliseconds). Thus, the response time of graph $G_{1}$ will be $r_{G_{1}}=O_{4}+r_{4}=210$, which is greater than $D_{G_{1}}=200$, hence the system is not schedulable.

\section{Scheduling and optimisation strategy}

Once we have a technique to determine if a system is schedulable, we can concentrate on optimising the total queue sizes. Our problem is to synthesise a system configuration $\psi$ such that the application is schedulable, i.e. the condition [Note 1]

$$
r_{G_{j}} \leq D_{G_{j}}, \quad \forall G_{j} \in \Gamma_{i}
$$

holds, and the total queue size $s_{\text {total }}$ is minimised [Note 2]:

$$
s_{\text {total }}=s_{\text {Out }}^{\text {CAN }}+s_{\text {Out }}^{\text {TTP }}+\sum_{\forall\left(N_{i} \in E T C\right)} s_{\text {Out }}^{N_{i}}
$$

Such an optimisation problem is NP-complete, hence obtaining the optimal solution is not feasible. We propose a resource optimisation strategy based on a hill-climb heuristic that uses an intelligent set of initial solutions in order to explore the design space efficiently.

\subsection{Scheduling and buffer optimisation heuristic}

The optimisation heuristic is outlined in Fig. 7. The basic idea of the OptimizeResources heuristic is to find, as a first step, a solution with the smallest possible response times, without considering the buffer sizes, in the hope of finding a schedulable system. This is achieved through the OptimizeSchedule function. Then, a hill-climbing heuristic iteratively performs moves intended to minimise the total buffer size while keeping the resultant system schedulable.

The OptimizeSchedule function outlined in Fig. 8 is a greedy approach that determines an ordering of the slots and their lengths, as well as priorities of messages and processes in the ETC, so that the degree of schedulability of the application is maximised. The degree of schedulability [10] is calculated as:

$$
\delta_{\Gamma}=\left\{\begin{array}{l}
f_{1}=\sum_{i=1}^{n} \max \left(0, R_{i}-D_{i}\right), \text { if } f_{1}>0 \\
f_{2}=\sum_{i=1}^{n}\left(R_{i}-D_{i}\right), \text { if } f_{1}=0
\end{array}\right.
$$

where $n$ is the number of process graphs in the application. If the application is not schedulable, the term $f_{1}$ will be positive, and in this case the cost function is equal to $f_{1}$. However, if the process set is schedulable, $f_{1}=0$ and we use $f_{2}$ as a cost function, as it is able to differentiate between two alternatives, both leading to a schedulable process set.

Note 1: The worst-case response time for a process graph $G_{i}$ is calculated based on its sink node as $r_{G_{i}}=O_{\text {sink }}+r_{\text {sink }}$. If local deadlines are imposed, they will also have to be tested in the schedulability condition.

Note 2: In the TTC, the synchronisation between processes and the TDMA bus configuration is solved through the proper synthesis of schedule tables, hence no output queues are needed. Input buffers on both TTC and ETC nodes are local to processes. There is one buffer per input message and each buffer can store one message instance (see explanation to Fig. 3).

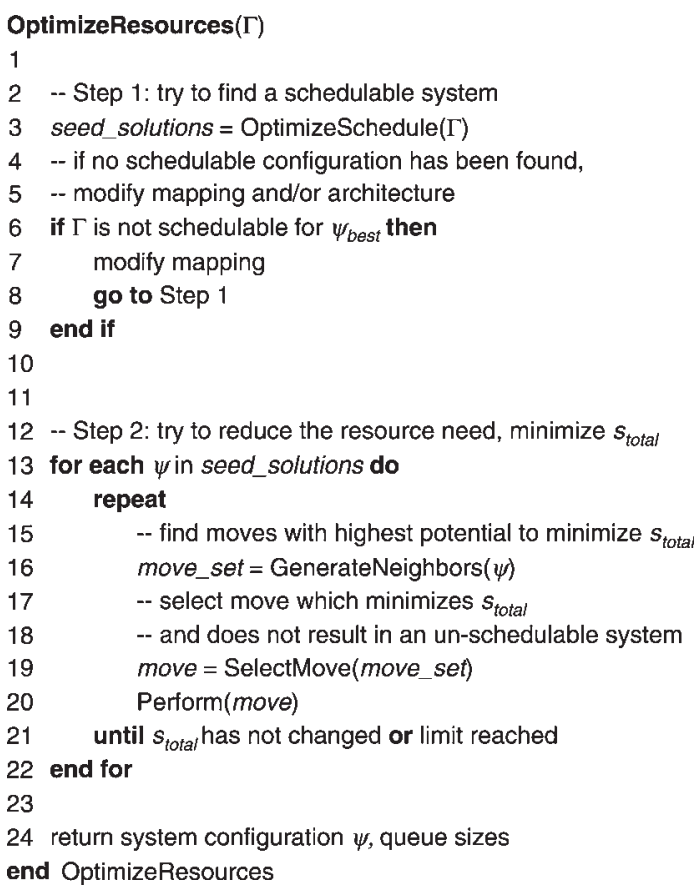

Fig. 7 OptimizeResources Algorithm

For a given set of optimisation parameters leading to a schedulable process set, a smaller $f_{2}$ means that we have improved the response times of the processes.

As an initial TTC bus configuration $\beta$, OptimizeSchedule assigns in order nodes to the slots and fixes the slot length to the minimal allowed value, which is equal to the length of the largest message generated by a process assigned to $N_{i}, \mathrm{~S}_{\mathrm{i}}=\left\langle\mathrm{N}_{\mathrm{i}}, \mathrm{size}_{\text {smallest }}\right\rangle$ (line 5 in Fig. 8). Then the algorithm starts with the first slot (line 8) and tries to find the node which, when transmitting in this slot, will maximise the degree of schedulability $\delta_{\Gamma}$ (lines 9-33).

Simultaneously with searching for the right node to be assigned to the slot, the algorithm looks for the optimal slot length. Once a node is selected for the first slot and a slot length fixed $\left(\mathrm{S}_{\mathrm{i}}=\mathrm{S}_{\text {best }}\right.$, line 32$)$, the algorithm continues with the next slots, trying to assign nodes (and to fix slot lengths) from those nodes that have not yet been assigned. When calculating the length of a certain slot we consider the feedback from the MulticlusterScheduling algorithm, which recommends slot sizes to be tried out. Before starting the actual optimisation process for the bus access scheme, a scheduling of the initial solution is performed, which generates the recommended slot lengths. We refer the reader to [9] for details concerning the generation of the recommended slot lengths.

In the OptimizeSchedule function the degree of schedulability $\delta_{\Gamma}$ is calculated based on the response times produced by the Multiclusterscheduling algorithm (line 18). For the priorities used in the response time calculation we use the 'heuristic optimised priority assignment' (HOPA) approach (line 15) from [18], where priorities for processes and messages in a distributed realtime system are determined, using knowledge of the factors that influence the timing behaviour, so that the degree of schedulability is improved.

The OptimizeSchedule function also records the best solutions in terms of $\delta_{\Gamma}$ and $s_{\text {total }}$ in the seed_solutions list in order to be used as the starting point for the second step of our OptimizeResources heuristic.

Once a schedulable system is obtained, our goal is to minimise the buffer space. Our design space exploration in 


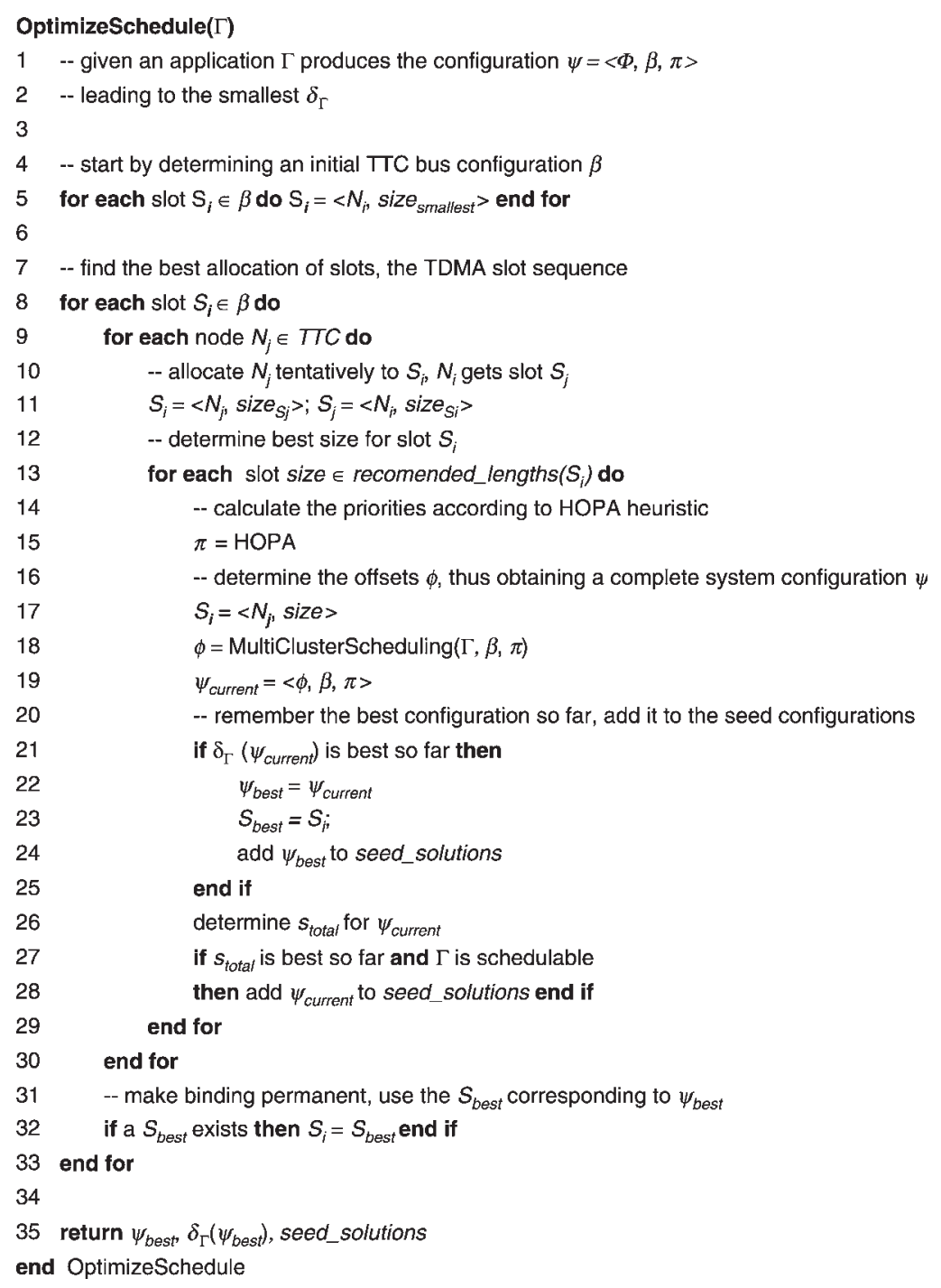

Fig. 8 OptimizeSchedule Algorithm

the second step of OptimizeResources (lines 12-22 in Fig. 7) is based on successive design transformations (generating the neighbours of a solution) called moves. For our heuristics, we consider the following types of moves:

- moving a process or a message belonging to the TTC inside its $[A S A P, A L A P]$ interval calculated based on the current values for the offsets and response times

- swapping the priorities of two messages transmitted on the ETC, or of two processes mapped on the ETC

- increasing or decreasing the size of a TDMA slot with a certain value

- swapping two slots inside a TDMA round.

The second step of the OptimizeResources heuristic starts from the seed solutions (line 13) produced in the previous step, and iteratively preforms moves to reduce the total buffer size, $s_{\text {total }}(12)$. The heuristic tries to improve on the total queue sizes, without producing unschedulable systems. The neighbours of the current solution are generated in the GenerateNeighbours functions (line 16 ), and the move with the smallest $s_{\text {total }}$ is selected using the SelectMove function (line 19). Finally the move is performed, and the loop reiterates. The iterative process ends when there is no improvement achieved on $s_{\text {total }}$, or a limit imposed on the number of iterations has been reached (line 21).
To improve the chances to find good values for $s_{\text {total }}$, the algorithm has to be executed several times, starting with a different initial solution. The intelligence of our OptimizeResources heuristic lies in the selection of the initial solutions, recorded in the seed_solutions list. The list is generated by the OptimizeSchedule function, which records the best solutions in terms of $\delta_{\Gamma}$ and $s_{\text {total }}$. Seeding the hill-climbing heuristic with several solutions of small $s_{\text {total }}$ will guarantee that the local optima are quickly found. However, during our experiments, we have observed that another good set of seed solutions are those that have a high degree of schedulability $\delta_{\Gamma}$. Starting from a highly schedulable system will permit more iterations until the system degrades to an unschedulable configuration, thus the exploration of the design space is more efficient.

\section{Experimental results}

For evaluation of our algorithms we first used process graphs generated for experimental purpose. We considered two-cluster architectures consisting of $2,4,6,8$ and 10 nodes, half on the TTC and the other half on the ETC, interconnected by a gateway. Forty processes were assigned to each node, resulting in applications of 80, 160, 240, 320 and 400 processes. Message sizes were randomly chosen between 8 and 32 bytes. Thirty examples were generated for 
each application dimension; thus a total of 150 applications were used for experimental evaluation. Worst-case execution times and message lengths were assigned randomly using both uniform and exponential distribution. All experiments were run on a Sun Ultra 10 computer.

To provide a basis for the evaluation of our heuristics, we have developed two simulated annealing (SA) based algorithms. Both are based on the moves presented in the previous section. The first one, named SA schedule (SAS), was set to preform moves such that $\delta_{\Gamma}$ is minimised. The second one, SA resources (SAR), uses $s_{\text {total }}$ as the cost function to be minimised. Very long and expensive runs have been performed with each of the SA algorithms, and the best ever solution produced has been considered to be close to the optimum value.

The first result concerns the ability of our heuristics to produce schedulable solutions. We have compared the degree of schedulability $\delta_{\Gamma}$ obtained from our OptimizeSchedule (OS) heuristic (Fig. 8) with the near-optimal values obtained by SAS. Figure 9 presents the average percentage deviation of the degree of schedulability produced by OS from the near-optimal values obtained with SAS. Together with OS, a straightforward approach (SF) is presented. For SF we considered a TTC bus configuration consisting of a straightforward ascending order of allocation of the nodes to the TDMA slots; the slot lengths were selected to accommodate the largest message sent by the respective node, and the scheduling has been performed by the Multiclusterscheduling algorithm (Fig. 5).

Figure 9 shows that, when considering the optimisation of the access to the communication channel, and of priorities, the degree of schedulability improves dramatically compared with the straightforward approach. The greedy heuristic OptimizeSchedule performs well for all the graph dimensions, having run-times that are more than two orders of magnitude smaller than with SAS. In the Figure, only the examples in which all the algorithms have obtained schedulable systems were presented. The SF approach failed to find a schedulable system in 26 out of the total 150 applications.

Next, we are interested in evaluating the heuristics for minimising the buffer sizes needed to run a schedulable application. We compare the total buffer need $s_{\text {total }}$ obtained by the OptimizeResources (OR) function with the near-optimal values obtained when using simulated annealing, this time with the cost function $s_{\text {total }}$. To find out how relevant the buffer optimisation problem is, we have compared these results with the $s_{\text {total }}$ obtained by the OS

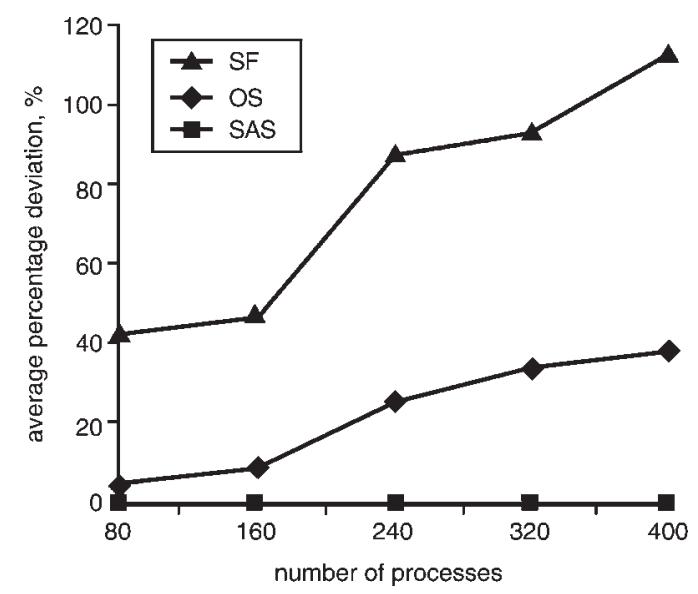

Fig. 9 Comparison of the scheduling optimisation heuristics

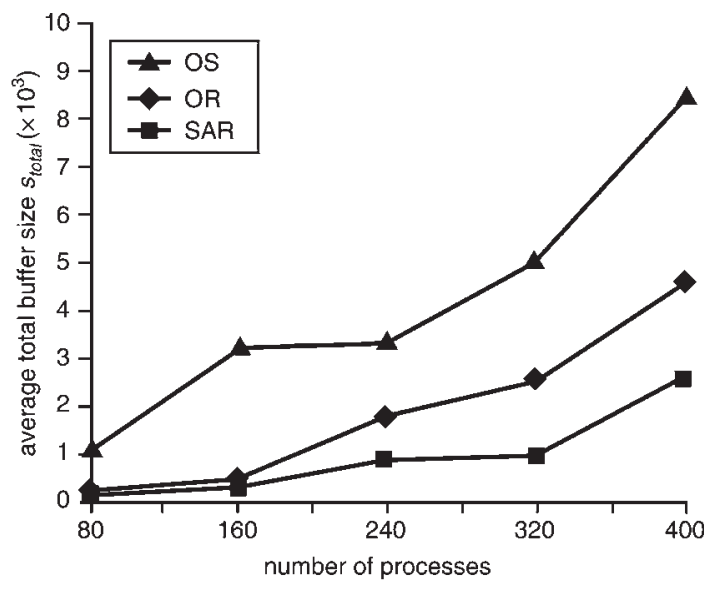

a

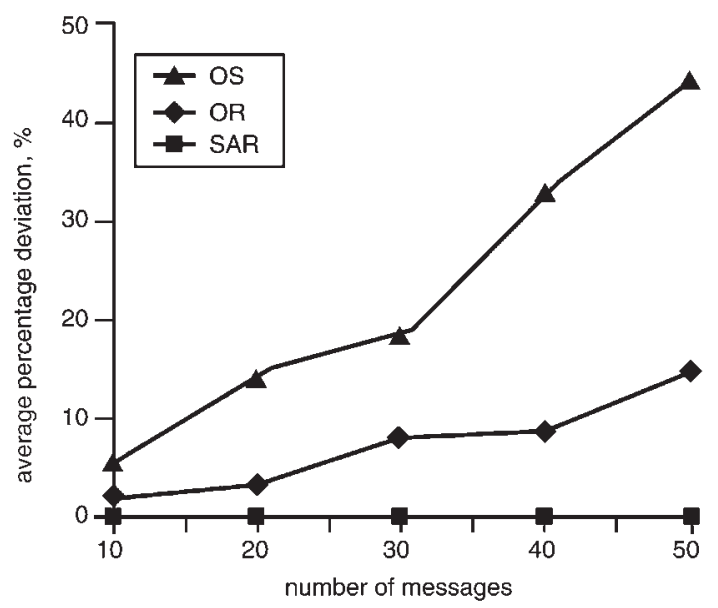

$b$

Fig. 10 Comparison of the buffer size minimisation heuristics

$a$ Bounds on total buffer size obtained with OS, OR, SAS

$b$ Percentage deviations for OS, OR from SAR

approach, which is concerned only with obtaining a schedulable system, without any other concern. As shown in Fig. 10a, OR is able to find schedulable systems with a buffer need half that of the solutions produced with OS. The quality of the solutions obtained by OR is also comparable with the one obtained with simulated annealing (SAR).

Another important aspect of our experiments was to determine the difficulty of resource minimisation as the number of messages exchanged over the gateway increases. For this, we generated applications of 160 processes with 10, 20, 30, 40 and 50 messages exchanged between the TTC and ETC clusters. Thirty applications were generated for each number of messages. Fig. $10 b$ shows the average percentage deviation of the buffer sizes obtained with OR and OS from the near-optimal results obtained by SAR. As the number of inter-cluster messages increases, the problem becomes more complex. The OS approach degrades very fast, in terms of buffer sizes, while the OR approach is able to find good quality results even for intense inter-cluster traffic.

When deciding on which heuristic to use for design space exploration or system synthesis, an important issue is the execution time. On average, our optimisation heuristics needed a couple of minutes to produce results, and hence they can be used inside a design space exploration loop. Although the simulated annealing approaches (SAS and SAR) had an execution time of up to three hours, they produced new-optimal results, and hence are suited for the final stages of the synthesis process. 
Finally, we considered a real-life example implementing a vehicle cruise controller. The process graph that models the cruise controller has 40 processes, and it was mapped on to an architecture consisting of a TTC and an ETC, each with two nodes, interconnected by a gateway. The 'speedup' part of the model has been mapped on the ETC while the other processes were mapped on the TTC. We considered one mode of operation with a deadline of $250 \mathrm{~ms}$. The straightforward approach SF produced an end-to-end response time of $320 \mathrm{~ms}$, greater than the deadline, while both the OS and SAS heuristics produced a schedulable system with a worst-case response time of $185 \mathrm{~ms}$. The total buffer need of the solution determined by OS was 1020 bytes. After optimisation with OR a still schedulable solution with a buffer need reduced by $24 \%$ has been generated, which is only $6 \%$ worse than the solution produced with SAR.

\section{Conclusions}

This paper has presented an approach to schedulability analysis for the synthesis of multi-cluster distributed embedded systems consisting of time-triggered and eventtriggered clusters, interconnected via gateways. The main contribution is the development of a schedulability analysis for such systems, including determining the worst-case queuing delays at the gateway and the bounds on the buffer size needed for running a schedulable system.

Optimisation heuristics for system synthesis have been proposed, together with simulated annealing approaches tuned to find near-optimal results. The first heuristic, OS, was concerned with obtaining a schedulable system by maximising the degree of schedulability. Our second heuristic, OR, was aimed at producing schedulable systems with a minimal required buffer size.

\section{Acknowledgment}

The authors are grateful to the industrial partners at Volvo Technological Development, Gothenburg, Sweden, for their close involvement and thoughtful feedback during this work.

\section{References}

1 Audsley, N.C., Burns, A., Davis, R.I., Tindell, K.W., and Wellings, A.J.: 'Fixed priority pre-emptive scheduling: an historical perspective', Real-Time Syst., 1995, 8, pp. 173-198

2 Balarin, F., Lavagno, L., Murthy, P., and Sangiovanni-Vincentelli, A.: 'Scheduling for embedded real-time systems', IEEE Des. Test Comput., 1998, 15, (1), pp. 71-82

3 Kopetz, H.: 'Real-time systems-design principles for distributed embedded applications' (Kluwer Academic Publishers, Norwell, MA, 1997)

4 Audsley, N., Tindell, K., and Burns, A.: 'The end of line for static cyclic scheduling?'. Proc. 5th Euromicro Workshop on Real-Time Systems, Oulu, Finland, 22-24 June 1993, pp. 36-41

$5 \mathrm{Xu}$, J., and Parnas, D.L.: 'On satisfying timing constraints in hard-realtime systems', IEEE Trans. Softw. Eng., 1993, 19, (1), pp. 70-84

6 'CAN Specification Version 2.0', Robert Bosch GmbH, 1991

7 Tindell, K., Burns, A., and Wellings, A.J.: 'Calculating CAN message response times', Control Eng. Pract., 1995, 3, (8), pp. 1163-1169

8 Tindell, K., and Clark, J.: 'Holistic schedulability analysis for distributed hard real-time systems', Microprocess. Microprogr., 1994, 50, (2-3), pp. 117-134

9 Eles, P., Doboli, A., Pop, P., and Peng, Z.: 'Scheduling with bus access optimization for distributed embedded systems', IEEE Trans. Very Large Scale Integr. (VLSI) Syst., 2000, 8, (5), pp. 472-491

10 Pop, P., Eles, P., and Peng, Z.: 'Bus access optimization for distributed embedded systems based on schedulability analysis'. Proc. Design, Automation and Test in Europe Conf., Paris, France, 27-30 March 2000, pp. 567-574

11 Lönn, H., and Axelsson, J.: 'A comparison of fixed-priority and static cyclic scheduling for distributed automotive control applications'. Proc. 11 th Euromicro Conf. on Real-Time Systems, York, UK, 9-11 June 1999, pp. $142-149$

12 'FlexRay Requirements Specification', http://www.flexray-group.com/

13 Pop, T., Eles, P., and Peng, Z.: 'Holistic scheduling and analysis of mixed time/event-triggered distributed embedded systems'. Int. Symp. on Hardware/Software Codesign, Estes Park, CO, 6-8 May 2002, pp. $187-192$

14 Pop, P., Eles, P., and Peng, Z.: 'Scheduling with optimized communication for time-triggered embedded systems'. Proc. 7th Int. Workshop on Hardware/Software Codesign, Rome, Italy, 3-5 May 1999, pp. $178-182$

15 Palencia, J.C., and González Harbour, M.: 'Schedulability analysis for tasks with static and dynamic offsets'. Proc. 19th IEEE Real-Time Systems Symp., Madrid, Spain, 2-4 December 1998, pp. 26-37

16 Yen, T.Y., and Wolf, W.: 'Hardware-software co-synthesis of distributed embedded systems' (Kluwer Academic Publishers, Norwell, MA, 1997)

17 Tindell, K.: 'Adding time-offsets to schedulability analysis'. Technical Report Number YCS-94-221, Department of Computer Science, University of York, 1994

18 Gutiérrez García, J.J., and González Harbour, M.: 'Optimized priority assignment for tasks and messages in distributed hard real-time systems'. Proc. 3rd Workshop on Parallel and Distributed Real-Time Systems, Santa Barbara, CA, April 1995, pp. 124-132 